\title{
Optoelectronic Sensor-based Shape Sensing Approach for Flexible Manipulators
}

\author{
Jia Han Benjamin Koh, Taegyun Jeong, Sangjin Han, Wanlin Li, Kawal Rhode, Yohan Noh, IEEE
}

Member

\begin{abstract}
This paper presents a novel two-axis shape sensor based on optical optoelectronic sensors and integrated with a flexible manipulator arm to measure the overall shape of the robotic arm. The disc-shape bio-compatible sensor presented here can be embedded as a sensing system into flexible manipulators and is applicable to the geometry of its structure and to the structure of any other similar multi-segment robotic manipulator. Design and calibration procedures of the device are introduced: experimental results allow defining a sensor matrix for real-time estimation of the pitch and roll of the plate above the sensor and confirms the usefulness of the proposed optical sensing approach.
\end{abstract}

\section{INTRODUCTION}

Minimally invasive surgery or MIS is a modern surgical technique which aims to reduce post-operative surgical trauma in patients relative to conventional surgical methods by reducing surgical incisions that are required to carry out operations. In order to carry out such a complex procedure, specialized robots have been designed to operate in such specialized conditions [1].

Robotic technology can provide valuable assistance and information for surgical procedures to surgeons [2]. For example, the da Vinci Surgical System is a surgical robot and control system for MIS. The robot arm of the da Vinci has multiple degrees of freedom (DoF), which makes it easier to carry out surgical operations in a restricted environment. The 3D camera of the da Vinci provides enlarged detailed visual information on the surgical site for the surgeon to view as they carry out the procedure. However, its robot arm has the following disadvantages: 1) the small workspace of its robot arm is constrained by rigid mechanical links in the robot arm outside of the patient's body; and 2) information describing

Manuscript received Feb $19^{\text {th }}, 2019$. The research leading to these results has received funding for Biomedical Engineering Project from the Department of Biomedical Engineering, School of Imaging Science and Biomedical Engineering, King's College London.

Jia Han Benjamin Koh, Kawal Rhode, Yohan Noh are with Department of Biomedical Engineering, School of Imaging Science and Biomedical Engineering, King's College London, UK (e-mail corresponding author: yohan.noh@kcl.ac.uk).

Taegyun Jeong is with the Department of Bioengineering, Imperial College London, UK.

Sangiin Han is with the Department of Electrical and Computer Engineering, Texas A\&M University, USA.

Wanlin Li is with the Electronic Engineering and Computer Science, Queen Mary University of London, UK. the interaction between the robot's arms and the patient's organs is not provided during the surgical procedure [3-4].

For these reasons, flexible manipulators including continuum robots have been adopted to assist surgeons in carrying out surgeries in the field of medicine. Choset et al. and Kahrs et al. have developed flexible manipulators which can control various shapes with high flexibility and high dexterity, using tendon driven mechanisms. [5 - 6] Webster III et al. created a concentric tube robot to reach any specific location of the brain without damage [7].

Although the surgical system with those flexible manipulators can allow accessing small openings or narrow spaces, and an imaging system at the head of the flexible manipulators that allow dexterous and precise navigation through difficult regions in the body, the surgical system is limited by the visual data it gives, as it only gives a point of view from the tip of the manipulator but not a view of the arm in the volume of the body or the shape of the arm as it moves through the body [8].

There are various conventional shape sensing mechanisms currently in use, with distinct advantage and disadvantages for surgical flexible manipulators. These shape sensing mechanisms include: Resistance-based approach, optical

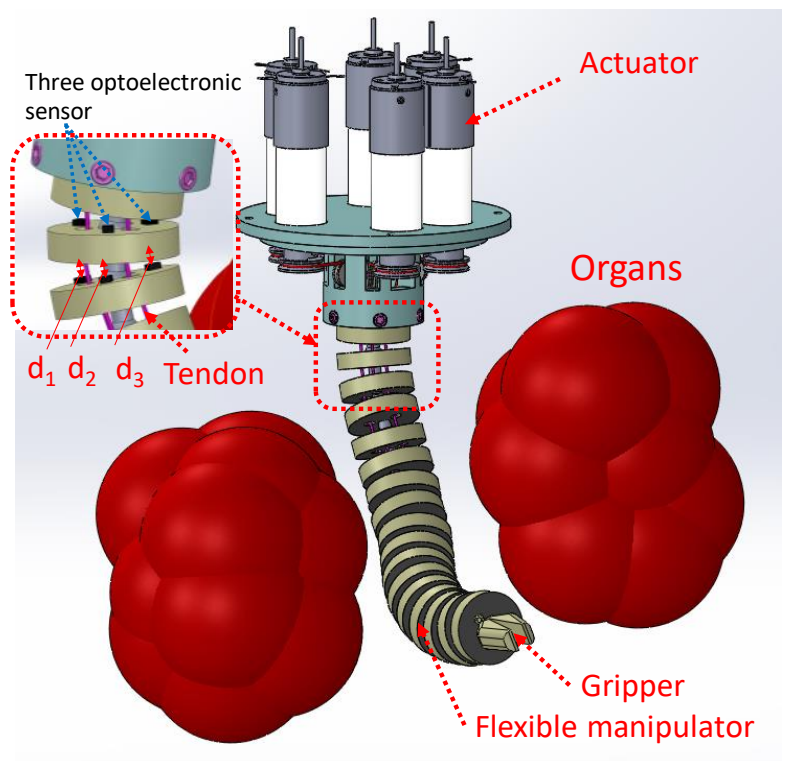

Figure 1. Overview of an optoelectronic sensors-based shape sensor with a flexible manipulator 
fibre-based approach and image-based approach. The resistance-based approach employs electro-conductive materials that vary in electrical properties proportionately with the strain experienced by the material, effectively acting as a strain gauge. An example of one of these strain gauges commonly used is conductive yarn, a thread-like metal wire wrapped around an elastic core. With an appropriate electrical supply and multiple conductive yarns, it can calculate the extent of the stretch experienced by the manipulator arm [6][9].

One optical fibre approach uses light intensity to detect shape. Light intensity modulated sensors use optical cables as light transmitters or both transmitter and receiver. As light is shone through the optical fibre, partial light modulation occurs through the wire, while the rest of the light is partially reflected to receiver cables from the reflectors. With multiple optical fibres, the varied reflected light intensities per cable would be able to provide the manipulator's orientation [7] [10].

The second optical fibre-based approach is based on Fibre Bragg Grating (FBG) sensing elements. This method uses fibre optic cables to be passed through the manipulator arm, with sensors positioned at intermediate points throughout the arm. As light travels through the bent manipulator arm, the wavelength of light changes proportional to the reflection experienced by the light as it travels through the deformed optical fibre along the arm. This change in wavelength is measured at intermediate parts of the arm and with two other cables in the manipulator arm you can find out the relative deformation of the manipulator. This approach has shown outstanding sensing modality, and it facilitates the overall manipulator's size to be super-miniaturised. Nevertheless, it is susceptible to temperature variation and the interrogator reading FBG sensing elements are very expensive [11-13]. Moreover, this approach cannot guarantee high-precision shape sensing based on the optoelectronic technology introduced in this paper.

An image-based approach uses imaging systems to interpret the shape of the manipulator. The two main methods are medical imaging modalities, which use fluoroscopic images to give better information of the shape through the use of multiple 2D images to approximate a 3D image, and imagers, which use cameras to gives point of view information from the tip of the surgical manipulator [8] [14-15].

Our proposed method would use a set of three optoelectronic sensors which can precisely measure three distances from one plate in the manipulator to the one above it. The relative two orientations (pitch and roll) between each of the two plates can be estimated using the voltage data from the optoelectronic sensors and linear regression. Then the position/orientation of the top of the flexible manipulator can be calculated by multiplying all the consecutive relative transformation matrices of every two plates of the flexible manipulator. This novel device takes advantage of the use of optoelectronic components that has a low power consumption, low level of noise and no need for any electronic filtering. The overall design should be easily added into existing flexible

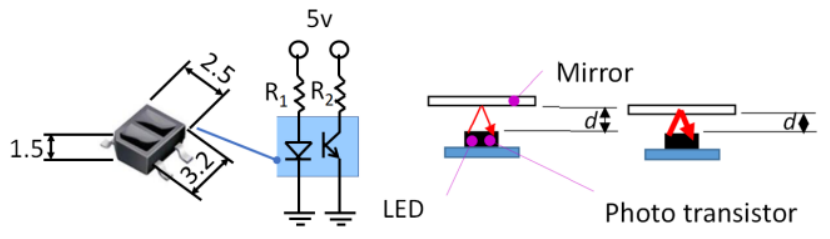

Figure 2. Optoelectronic sensor QRE1113 (Fairchild Semiconductor Corp.) and distance measurement principle
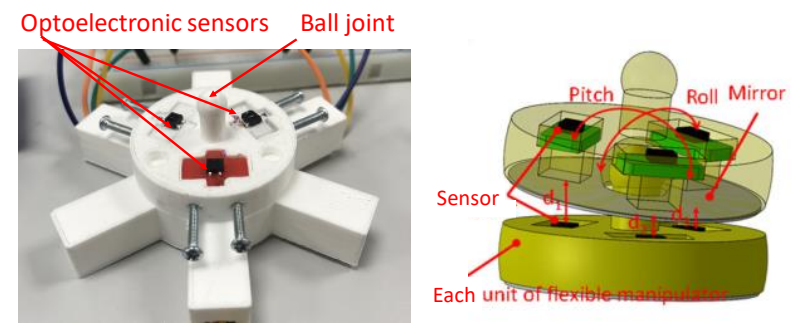

Figure 3. Shape sensing principle based on the three optoelectronic sensors and ball joint mechanism.

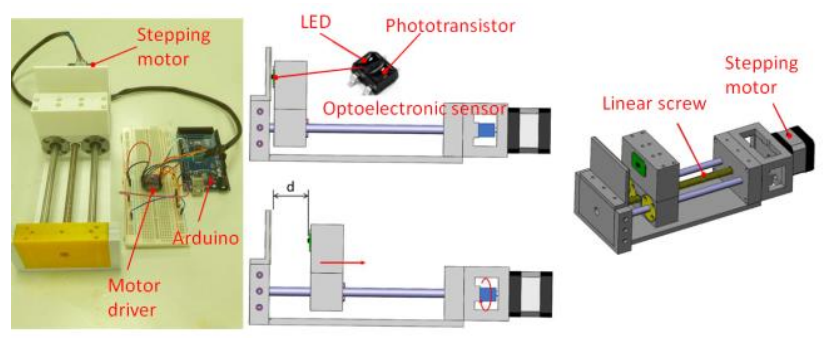

Figure 4. Experiment setup for optimising two resistors

manipulator designs built based on tendon driven mechanisms.

In this paper, we focus on the development of a shape sensing sensor for the flexible manipulators and show how to design and calibrate a transformation matrix by considering integration of the sensor with its manipulator.

\section{DESIGN METHODS AND FABRICATION}

\section{A. Design Concept}

The design concept of the shape sensor should satisfy several conditions, which are:

1) The sensor device should be integrated into the flexible manipulator's design, being embedded in every plate to be able to determine the transformation matrix.

2) The sensor must be able to measure flexure of the overall manipulator arm in any direction as well as any complex shapes producible by the manipulator i.e. "S" shape.

3) The sensor should be able to measure at least 2 axes of rotations (roll and pitch). Although measuring the third axis of rotation (yaw) is possible, priority is given to the roll and pitch as yaw can be easily constrained in manipulator designs.

In this paper, the proposed shape sensing is supposed to be integrated into flexible manipulators of medical instruments, and its diameter should be less than $15 \mathrm{~mm}$ such that it can be inserted through the maximum size of trocar. However, in this paper, in order to prove the sensing algorithm, a bigger size of 
the shape sensing will be described.

\section{B. Configuration of Shape Sensor and Sensing Principle}

The shape sensor consists of three optoelectronic sensors (model QRE1113 from Fairchild Semiconductor Corp.) and a circular plate structure with three indents for each optoelectronic sensor which is made from true white PLA (polylactic acid) plastic and designed with an FDM (fused deposition modelling) 3D rapid prototyping machine. The structure design is based on the flexible manipulator being comprised of a large number of these plates, linked together with ball and socket joints with three spring between each plate to restrict the yaw rotation as shown in Figures 1 and 2.

The optoelectronic sensors are comprised of a photo diode and a phototransistor which emit and receive a specific range of wavelengths of light respectively (Figure 2). The closer the distance between the current plate and the plate above it, the higher the intensity of the reflected light that is received by the phototransistor (Figure 3). The amount of light reflected is inversely proportional to the voltage output of the optoelectronic sensor. From this output voltage, the distance between the optoelectronic sensor and the plate above can be determined. When the flexible manipulator is deformed in anyway, each optoelectronic sensor found in each plate of the manipulator would be able to measure the distance between plates at three points (Figures 1 and 3). Using the three measures of distance, the roll and pitch of the plate above can be calculated. Calculation of the roll and pitch of every subsequent plate would then be able to calculate the shape and the distal position of the flexible manipulator by multiplying consecutive relative transformation matrices as shown in Eq. (1).

\section{CALIBRATION AND OPTIMISATION OF OPTOELECTRONIC SENSOR}

An optoelectronic sensor can measure small and large distances precisely. Its output voltages with respect to distances have a linear curve, and without an amplifier, a large voltage variation can be obtained with respect to distance changes. In this section, optimising the two resistors to measure a large range of distances by the optoelectronic sensor will be described as below:

\section{A. Experiment Setup}

1) Experiment device for optimising resistors for an optoelectronic sensor

The device aims at optimising the two resistors combination for the optoelectronic sensor such that the range of distances we want to measure has a distinct relationship to the output voltage of the optoelectronic sensors. The device consists of a stepper motor with an optoelectronic sensor attached to an arm and a white reflective board. When activated, the stepper motor would turn such that the arm pushes the optoelectronic sensor towards the reflective board over a set distance (Figure 4). The calibration is repeated for different combinations of resistors for both the LED and the

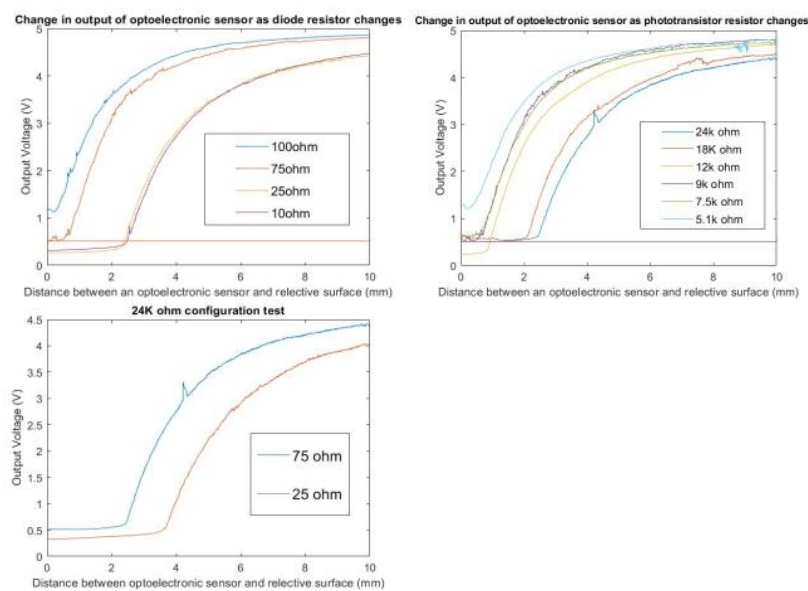

Figure 5. Comparisons of different resistor configurations.

$$
\boldsymbol{T}_{0,5}=\boldsymbol{T}_{0,1} \times \boldsymbol{T}_{1,2} \times \boldsymbol{T}_{2,3} \times \boldsymbol{T}_{3,4} \times \boldsymbol{T}_{4,5}
$$

phototransistor as seen in Figure 2.

Based on design measurements, the optoelectronic sensor must be able to produce a distinct relationship for the distance between the optoelectronic sensor and reflective surface and the output voltage of the optoelectronic sensors. This therefore allows us to be able to measure the range of movements produced from the pitch and roll of the plate above the sensors. For our case, the optoelectronic sensor must be able to function within $2 \mathrm{~mm}$ to $8 \mathrm{~mm}$ as those are the minimum and maximum distance between the plates during the full range of motion.

\section{2) Experiment device for optimising resistors for an} optoelectronic sensor

Due to the design of the plates, the maximum and minimum possible distances separating the top and bottom plates is 2 $\mathrm{mm}$ to $8 \mathrm{~mm}$. With this in mind, we aimed for the resistors to be able to produce distinct values for that range. As seen from Figure 5, the shape of the curve for output voltage is a negative exponential decay to a steady state value of 4.88 Volts. Because of this, we would want the part of the curve with the largest changes in value to be in the desired range. The circuit
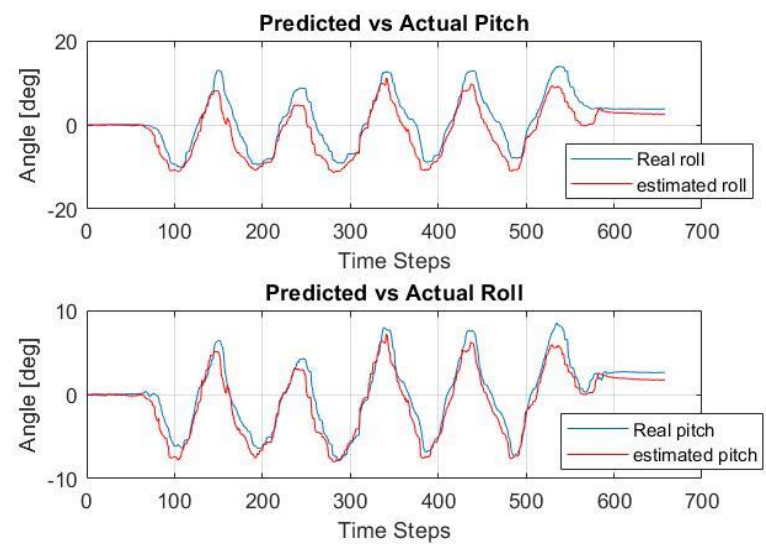

Figure 6. Comparison between real and estimated roll values. 
diagram with $R_{1}$ and $R_{2}$ can be seen from Figure 2. The top left graph in Figure 5 shows the relationship in the effective range of the optoelectronic sensor when the value of $R_{1}$ is varied while $R_{2}$ is kept constant. A similar experiment is conducted with $R_{2}$ being changed while $R_{1}$ is kept constant (Figure 5, top right). Therefore, the choice of a $75 \Omega$ resistor and a $24 \mathrm{k} \Omega$ resistor is chosen for $R_{1}$ and $R_{2}$ respectively (Figure 5, bottom left).

\section{3) Calibration for pitch and roll}

The device aims to calibrate the roll and pitch relative to the three optoelectronic sensor output voltages by finding the transformation matrix that governs the three inputs with the two separate outputs. The top plate of the device houses the Inertial Measurement Unit or IMU sensor (LPMS-B sensor, Life Performance Research, Tokyo, JAPAN) that measures roll and pitch of the plate and has a smooth white surface on the underside for the optoelectronic sensor to reflect off. The orientation trial is carried out by locking the two antiparallel arms with the blue clips as seen in Figure 7 and 'see-sawing' the top plate slowly across its antiparallel arms ( 1 and 4, 2 and 5,3 and 6) while both the IMU on the plate as well as the optoelectronic sensors record data on roll and pitch of the top plate and output voltage respectively.

\section{4) Calibration for pitch and roll of multiple segments}

The transformation matrix is unique for each segment and is calculated by calibrating each segment on its own first before the full experiment is carried out. This is done by restricting non-essential segment movement by attaching rings between them, then carrying out the same calibration steps as stated in point III part 3 and seen in Figure 7.

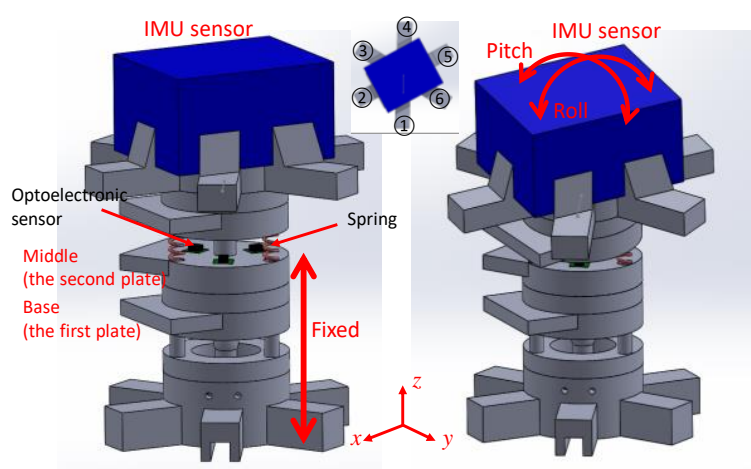

Figure 7. Calibration example of the second segment of the four-segment experimental set up

TABLE I. EXPERIMEMTAL CONFIGURATION OF THE APPLIED PITCH AND ROLL FOR CALIBRATION

\begin{tabular}{|c|c|c|}
\hline $\begin{array}{c}\text { Position on } \\
\text { Calibration Base } \\
\text { (Fig. 9) }\end{array}$ & $\begin{array}{c}\text { Pitch and Roll } \\
\text { Components }\end{array}$ & $\begin{array}{c}\text { Angle } \\
\text { Ranges }\end{array}$ \\
\hline (1) to 4 & Pitch & -15 to 15 Degrees \\
\hline (2) to 5) & Roll and Pitch & $\begin{array}{c}-12 \text { to } 12 /-10 \text { to } 10 \\
\text { Degrees }\end{array}$ \\
\hline (3) to 6) & Roll and Pitch & $\begin{array}{c}-12 \text { to } 12 /-12 \text { to } 6 \\
\text { Degrees }\end{array}$ \\
\hline
\end{tabular}
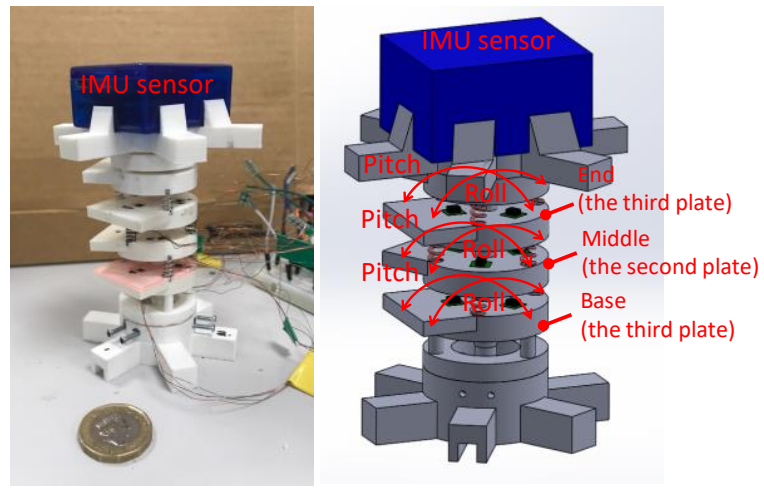

Figure 8. Design of multi-segment sensor calibration device.

\section{B. Calibration of model equation by linear regression}

Linear regression (LR) finds a relationship between two or more independent variables and a dependent variable by fitting a linear equation to the observed data set. This produces a matrix with effective weights for the magnitude of how much each of the independent variables affect the dependent variable.

The calibration data in every calibration test is used to generate a transformation matrix that models the relationship between the three input voltages from each optoelectronic sensor with the physical values of roll and pitch angles of the plate above the optoelectronic sensors. The equation for each segment would therefore be governed by Eq. (2). Eq. (3), (4) and (5) are the transformation matrices calculated from the calibration of each individual plate in point III part 3.

$$
\begin{gathered}
{\left[\begin{array}{lll}
k_{11} & k_{12} & k_{13} \\
k_{21} & k_{22} & k_{23}
\end{array}\right] *\left[\begin{array}{l}
v_{1} \\
v_{2} \\
v_{3}
\end{array}\right]=\left[\begin{array}{c}
\text { Pitch } \\
\text { Roll }
\end{array}\right]} \\
k_{\text {plate 1 }}=\left[\begin{array}{ccc}
-9.1724 & 14.5129 & 0.3462 \\
1.9257 & -10.2038 & -8.2922
\end{array}\right] \\
k_{\text {plate 2 }}=\left[\begin{array}{ccc}
-9.3889 & 2.7441 & 5.7697 \\
-6.0725 & 10.3538 & -3.6872
\end{array}\right] \\
k_{\text {plate 3 }}=\left[\begin{array}{ccc}
4.2860 & -1.1503 & -7.6587 \\
10.9468 & -9.6748 & 4.2994
\end{array}\right]
\end{gathered}
$$

\section{COMPARISON BETWEEN SHAPE SENSOR AND IMU SENSOR}

After calibration, a comparison test for the multiple segments is conducted by having all three plates be move and the motion will be recorded by the IMU sensor. This setup can be seen in Figure 8.

Once the three transformation matrices are calculated, the resultant angles of pitch and roll for each segment are summed together and both the final pitch and roll of the model as well as the pitch and roll recorded by the IMU sensor is offset by their initial values so that all values start from zero. 

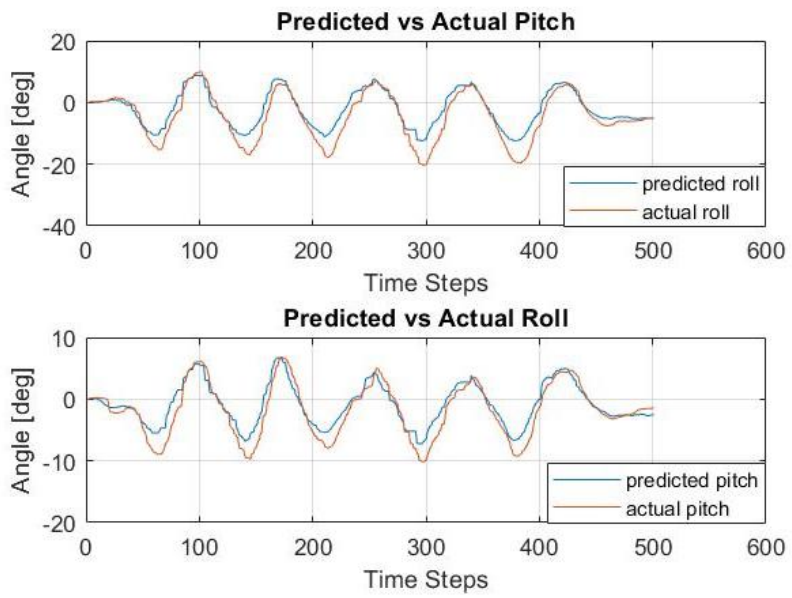

Figure 9. Comparison between three segment pitch and roll and actual pitch and roll.

TABLE II. SENSOR ERRor Property For Single Plate

\begin{tabular}{|c|c|c|c|}
\hline \multirow{2}{*}{ Angle } & $\begin{array}{c}\text { Calculated } \\
\text { Angle }\end{array}$ & $\begin{array}{c}\text { Maximum } \\
\text { Error }\end{array}$ & $\begin{array}{c}\text { Percentage } \\
\text { Error [\%] }\end{array}$ \\
\cline { 2 - 4 } & Degrees & Degrees & {$[\%]$} \\
\hline Roll & \pm 11.3 & 8.9 & 39.4 \\
\hline Pitch & \pm 8.0 & 3.7 & 23.1 \\
\hline
\end{tabular}

TABLE III. SENSOR ERROR PROPERTY FOR THREE PLATES

\begin{tabular}{|c|c|c|c|}
\hline \multirow{2}{*}{ Angle } & $\begin{array}{c}\text { Calculated } \\
\text { Angle }\end{array}$ & $\begin{array}{c}\text { Maximum } \\
\text { Error }\end{array}$ & $\begin{array}{c}\text { Percentage } \\
\text { Error [\%] }\end{array}$ \\
\cline { 2 - 4 } & Degrees & Degrees & {$[\%]$} \\
\hline Roll & \pm 26.6 & 18.0 & 33.8 \\
\hline Pitch & \pm 22.9 & 9.7 & 21.2 \\
\hline
\end{tabular}

As shown in Eq. (2), from the sensor voltage samples such as $V_{l}, V_{2}$, and $V_{3}$, the estimated pitch and roll are obtained by means of the calculated transformation matrix. However, the estimated pitch and roll have errors in comparison with the actual pitch and roll as shown in Figures 6 and 9. Nevertheless, the calibration matrix can estimate the pitch and roll within a sensor range error with a maximum absolute and percentage error as shown in Figures 6 and 9 and Table II and III. With the measurement of the pitch and roll of the plates, this can be translated into the pitch and roll of the flexible manipulator and proves to be an effective shape sensor.

\section{CONCLUSION AND FUTURE WORKS}

With this paper, we have presented a shape sensing sensor using optoelectronic sensors to measure the distance between each plate at three points to measure the orientation of the plate above, with the summation of multiple plate orientations to give the full shape of the flexible manipulator. The sensor design enables a multi-plate snake-like design that can be a novel flexible manipulator design. We believe that the demonstrated design and solution can be applied to other similar flexible manipulators which employ multiple elements via rigid joints. Finally, we validated the sensor range through experimental measurements.
In future work, sensor characteristics such as the effect of yaw on the shape sensing linear model, usage of non-linear regression to form a more accurate transformation matrix, reducing errors, repeatability and crosstalk will be evaluated and improved. Additionally, the sensors will have actuators that can control and manipulate the entire flexible manipulator with cables running through various segments of the manipulator as well as an OpenGL C++ program to display and give real time feedback on the full flexible manipulator shape during surgery.

\section{REFERENCES}

[1] J. L. Ochsner, "Minimally Invasive Surgical Procedures," Ochsner J., vol. 2, no. 3, pp. 135-136, Jul. 2000.

[2] T.L.Ghezzi, O.C.Corleta, "30 Years of Robotic Surgery, "Springer Link. [Online] https://link.springer.com/article/10.1007\%2Fs00268-016-3543-9.

[3] N. Simaan, K. Xu, A. Kapoor, W. Wei, P. Kazanzides, P. Flint, and R. Taylor, "A System for Minimally Invasive Surgery in the Throat and Upper Airways," Int. J. Robotics Research (special issue on medical robotics), vol. 28- 9, pp. 1134-1153, 2009.

[4] H. Ashrafian, O. Clancy, V. Grover, and A. Darzi, "The evolution of robotic surgery: surgical and anaesthetic aspects," BJA Br. J. Anaesth., vol. 119, no. suppl_1, pp. i72-i84, 2017.

[5] A.T. Hillel, A. Kapoor, N. Simaan, R.H. Taylor, P. Flint, "Applications of Robotics for Laryngeal Surgery," Laryngeal Cancer-Otolaryngologic Clinics of North America, Nasir Bhatti \& Ralph P. Tufano Eds., vol. 41, no. 4, pp. 781-791, 2008.

[6] H. A. Wurdemann, S. Sareh, A. Shafti, Y. Noh, A. Faragasso, D, S. Chathuranga, H. Liu, S. Hirai, K. Althoefer, "Embedded electro-conductive yarn for shape sensing of soft robotic manipulators," in 2015 37th Annual International Conference of the IEEE Engineering in Medicine and Biology Society (EMBC), pp. 8026-8029, 2015.

[7] S. Sareh, Y. Noh, M. Li, T. Ranzani, H. Liu, K. Althoefer, "Macrobend optical sensing for pose measurement in soft robot arms," Smart Materials and Structures, vol 24, no 12, 2015.

[8] A. Vandini, C. Bergeles, F. Lin, G. Yang, "Vision-Based Intraoperative Shape Sensing of Concentric Tube Robots," 2015 IEEE/RSJ International Conference on Intelligent Robots and Systems (IROS), 2015.

[9] A-V. Ho, S. Hirai, "Slip perception via soft robotic skin made of electroconductive yarn," Mechanics of Localized Slippage in Tactile Sensing, pp 113-154, 2014.

[10] T. C. Searle, K. Althoefer, L. Seneviratne, H. Liu, "An Optical Curvature Sensor for Flexible Manipulators", The 2012 IEEE International Conference on Robotics and Automation (ICRA 2012), pp. 4401-4405, 2012.

[11] M. Perry, P. Orr, P. Niewczas, M. Johnson. "High-Speed Interferometric FBG Interrogator With Dynamic and Absolute Wavelength Measurement Capability," Journal of Lightwave Technology, vol. 31, no. 17, pp 2897-2903, 2013.

[12] M. Lei, W. Zou, X. Li, J. Chen. "Ultrafast FBG Interrogator Based on Time-Stretch Method," IEEE photonics Technology Letters, vol. 28, no. 7, pp 778-781, 2016.

[13] M. A. Davis, A. D. Kersey, J. Sirkis, E. J. Friebele, "Shape and vibration mode sensing using a fibre optic Bragg grating array," Smart Materials and Structures, pp 759-765, 1996.

[14] C. Delmas, M. Berger, E. Kerrien, C. Riddel, Y. Trousset, R. Anxionnat, S. Bracard, "Three-dimensional curvilinear device reconstruction from two fluoroscopic views," SPIE. Medical Imaging 2015: Image- Guided procedures, robotics interventions, and modelling, pp. 94150F, 2015.

[15] C. Shi, X. Luo, P. Qi, T. Li, S. Song, Z. Najdovski, T. Fukuda, H. Ren, "Shape Sensing Techniques for Continuum Robots in Minimally Invasive Surgery: A Survey," IEEE Transactions on Biomedical Engineering, vol. 64, no. 8, pp. 1665 - 1678, 2017 